\title{
Regula Forster, Wissensvermittlung im Gespräch. Eine Studie zu klassisch- arabischen Dialogen (Islamic History and Civilization 149), Leiden, Boston: Brill, 2017, xii+532, ISBN 978-90-04-32670-5
}

Besprochen von Elvira Wakelnig, Universität Wien, Wien, Österreich, elvira.wakelnig@univie.ac.at

https://doi.org/10.1515/islam-2020-0015

Die vorliegende Monographie, bei der es sich um die überarbeitete Version der Habilitationsschrift von Regula FORSTER handelt, beschäftigt sich mit einer bisher in der Forschung vernachlässigten Textgattung der klassisch-arabischen Literatur, dem Dialog. Wenn die Verfasserin ihren Band mit einem Zitat von Vittorio HösLE beginnt, nach dem „In der islamischen Welt ... die Dialogform nur selten verwendet wird“ (1), muss sie es sich gefallen lassen, dass man ihr polemisch mit einem Zitat von Rina DRORY antwortet, das die völlig entgegengesetzte Einschätzung zum Ausdruck bringt und von ,the dominance of dialogue, rather than narration, in the canonized literary repertoire of prose writing (e.g. $a d a b$...) “ spricht, vor allem da der - zugegebenermaßen äußerst kurze Eintrag Drorys „dialogue in literature, medieval“ in der Encyclopedia of Arabic Literature I, hg. v. Julie Scott Meisami und Paul Starkey, 1998, S. 191, aus dem das Zitat stammt, in der gesamten Monographie mit keinem Wort erwähnt wird. Aber selbstverständlich verweist auch Regula FoRSTER selbst nach der Anführung von HöSLEs Zitat sogleich auf die Omnipräsenz des Dialogs in der klassisch-arabischen Literatur (1, Fußnote 2). Sodann gibt die Verfasserin zwecks Rechtfertigung ihrer Verwendung des anachronistischen Begriffes Dialog „im Sinn der schriftlichen Darstellung einer mündlichen Interaktion“ (4) zunächst einen Überblick über die tatsächlich vorhandene klassisch-arabische Terminologie. Hierbei zeigt sich, dass Termini wie munāzara und das synonyme muğādala zu weit gefasst sind, da sie Auseinandersetzungen zwischen Vertretern unterschiedlicher Standpunkte genauso umfassen können wie Monologformen (ibid.). Um ihr Textkorpus abzugrenzen, gibt die Verfasserin eine präzise Charakterisierung der aufgenommenen Dialoge: diese Dialoge weisen mindestens drei Sprechwechsel und einen Minimalumfang von drei Druckseiten auf (3) und haben außerdem eine wissensvermittelnde Funktion, d.h. sie stellen den Wissenserwerb von Gesprächspartnern und den Ablauf des Lehrens und Lernens dar (5). Als zeitlicher Rahmen wird die Periode zwischen dem achten und der Mitte des elften Jahrhunderts gewählt (7). Interreligiöse Dialoge sowie Texte, die sich an eine kleine Gruppe von Eingeweihten wenden, werden ausdrücklich ausgeschlossen (17f.). So entsteht ein Korpus von 42 Dialogen, die am Ende des Buches in einem Repertorium genannten Kapitel (429-480) ausführlich dargestellt werden. So werden Autor, Datie- 
rung und Inhalt jedes Textes besprochen, sowie Angaben zu benutzten Editionen bzw. Handschriften, vorhandenen modernen Übersetzungen und ausgewählter Sekundärliteratur gemacht. Die kurze Beschreibung des Textkorpus am Anfang des vorliegenden Bandes (14-20) fällt hingegen sehr kurz aus. Hier wäre eine etwas ausführlichere Darstellung der einzelnen Dialoge für die Lektüre sicherlich hilfreich gewesen. Außerdem wäre eine Übersetzung der arabischen Titel allen des Arabischen nicht Kundigen sicher entgegengekommen.

Zwecks Analyse werden die Dialoge des Korpus in folgenden sechs Gruppen zusammengefasst: 1. religiöse Dialoge, bei denen weiters zwischen (proto-) sunnitischen und schiitischen Texten unterschieden wird; 2. Dialoge mit Gott; 3. Dialoge aus narrativen Texten; 4. Dialoge über Geschichte und 5. über Grammatik; sowie 6. natur- und geheimwissenschaftliche Dialoge.

$\mathrm{Ob}$ es der derzeitige Wissenschaftsbetrieb mit seiner Verpflichtung zur genauen Angabe der verwendeten Methodik nicht etwas zu weit treibt, mögen sich Lesende fragen, wenn sie zweimal daraufhin gewiesen werden, dass das Textkorpus einem „Close Reading“ unterzogen wird (7 u. 8) - dieses sollte sich von selbst verstehen. In einem ersten Teil (22-191) werden zunächst die äußeren Bedingungen bzw. die Grundlagen der Dialoge untersucht. So wird die Redekonstellationstypik in ihren verschiedenen Aspekten, als da sind die Dialogpartner, ihre Zahl und ihr gegenseitiges Verhältnis, aufgezeigt sowie der zeitliche und örtliche Rahmen, der Anlass der Dialoge und die Rolle des Erzählers (7). In einem zweiten Teil (192-419) wird die innere Gestaltung der Dialoge und deren Gesprächsführung analysiert (7f.).

Die Lektüre dieser beiden großen, die eigentliche Analyse ausmachenden Teile gestaltet sich etwas beschwerlich, da die besprochenen Elemente wie Dialogteilnehmer, Zahl der Dialogteilnehmer, Argumentationsweisen und Emotionen jeweils hintereinander für alle sechs Dialoggruppen abgehandelt und anschließend nochmals zusammengefasst werden. Die Ergebnisse, die sich aus der Untersuchung der religiösen Dialoge ergeben, werden dabei sogar zweimal resümiert, zuerst nach den separaten, aufeinanderfolgenden Darstellungen der sunnitischen und der schiitischen Dialoge sowie in der allgemeinen Zusammenfassung am jeweiligen Kapitelende. Zudem werden die Resultate der beiden Teile an deren Ende nochmals resümiert. Diese Vorgangsweise führt zu allzu häufigen Wiederholungen. Außerdem müssen sich die Lesenden mit den einzelnen Dialogen aufgrund der Angaben im Repertorium ausreichend vertraut gemacht haben und diese Angaben auch während der gesamten Lektüre geistig bereithalten (was sich bei 42 Texten schwierig gestaltet), um den Darlegungen gut folgen zu können. Eine andere Struktur hätte das Lesen gewiss erleichtert. In der vorliegenden Form eignet sich die Monographie weniger zur durchgehenden Lektüre als zum punktuellen Nachschlagen des einen oder anderen Aspekts. 
Das Fazit (420-428) gibt einen guten Überblick über die erreichten Einsichten sowie über die noch offenen Fragestellungen bei der Erforschung des klassisch-arabischen Dialogs (427f.). So hat die Verfasserin die zuweilen geäußerte Meinung, es handle sich bei Dialogen um eine „dialogisierte Abhandlung“, widerlegen können (420). Im Gegensatz dazu ist gezeigt worden, dass sich Dialoge dazu eigenen, den im besten Falle mündlich stattfindenden Vorgang des Unterrichts, der Wissensvermittlung und des Wissenserwerbs nachzubilden und so dem im untersuchten Zeitraum vorherrschenden „Ideal der Mündlichkeit“ einigermaßen gerecht zu werden (ibid.). Hierzu passend hat sich zudem ergeben, dass es zumeist der an Kenntnissen unterlegene Gesprächspartner ist, der die Geschwindigkeit der Wissensvermittlung bestimmt (424). Somit geben Dialoge eine realistische Gesprächssituation wieder. Dialoge tragen weiters zur Verortung der Lesenden in einer bestimmten Gruppe bei und fördern deren Abgrenzung von Anderen, indem sie ihnen ein Identifikationsangebot mit dem Protagonisten machen (6 u. 421). Die Analyse der Verfasserin hat gezeigt, dass der Ort in den untersuchten Texten eine viel wichtigere Komponente ist als die Zeit und dass sich der in jedem Fall vorhandene Erzähler im eigentlichen Dialogverlauf zurückhält. Ebenso lässt sich an den analysierten Dialogen der Einsatz von Macht für den Wissenserwerb und für die Bestimmung der Art des vermittelten Wissens nachvollziehen (423). Während Emotionen keine große Rolle bei der Gestaltung des Gesprächsverlaufs zukommt, werden Schwüre, Flüche, Gebete und jegliche Bezugnahmen auf Gott bewusst eingesetzt, etwa um inhaltlich zentrale Stellen zu kennzeichnen (425f.).

Der Band schließt mit einem Personen- und Ortsregister (511-519), einem Verzeichnis der Buchtitel (520-523), einem Sachregister (524-531) und einem Verzeichnis der behandelten Koranstellen (532) ab.

Wissensvermittlung im Gespräch bietet eine umfassende Behandlung von 42 klassisch-arabischen Dialogen, die in Hinblick auf eine Vielzahl von Elementen der inneren und äußeren Gesprächsgestaltung untersucht werden. Die einzelnen Punkte lassen sich innerhalb der Monographie leicht auffinden und sind auch als Einzelkapitel verständlich. Die unternommene Analyse hat zu wichtigen Ergebnissen für die klassisch-arabische Dialogforschung geführt. Darüber hinaus werden zukünftige Fragestellungen formuliert. Somit liegt hier ein wichtiger Beitrag zur Erforschung der Form des Dialoges und der Art und Weise der Wissensvermittlung in der klassisch-arabischen Literatur vor. 